\title{
THE DESORPTION BEHAVIOUR OF IMPLANTED NOBLE GASES AT LOW ENERGY ON SIIICON SURFACES *
}

\author{
A.H.M. HOLTSLAG and A. VAN SILFHOUT \\ Department of Applied Physics, Twente University of Technology, P.O. Box 217, \\ 7500 AE Enschede, The Netherlands
}

Received 17 February 1987; accepted for publication 15 April 1987

Under UHV conditions, clean crystalline Si(111) surfaces have been bombarded mass-selectively at room temperature with noble gas ions, $\mathrm{Ne}^{+}, \mathrm{Ar}^{+}, \mathrm{Kr}^{+}$, at normal incidence. By means of stepwise heating up to $1050 \mathrm{~K}$ the activation energies and desorbed doses of the noble gases have been straight forwardly determined as a function of temperature and bombardment dose. Firstly a short review of the kinetics of desorbed adsorbates is given and compared with a simple model for the desorption of particles embedded in a surface layer. This shows that the rate constant $\nu$ in the latter case is orders of magnitude lower and are in agreement with obtained experimental values. From the obtained activation energies we have concluded that these values have to be explained by the silicon properties and that the influence of the noble gas is of second-order importance. The present method enables the simultaneous measurement of both the ellipsometric and desorption spectra during stepwise heating of the sample.

\section{Introduction}

This paper reports the results and interpretation of desorption measurements carried out on clean $\mathrm{Si}(111)$ surfaces mass-selectively bombarded with noble gas ions, $\mathrm{Ne}^{+}, \mathrm{Ar}^{+}, \mathrm{Kr}^{+}$, at normal incidence with an acceleration voltage of $2.2 \mathrm{kV}$.

From the desorption measurements we would like to obtain information about the concentrations of the implanted ions, the type of defects and the desorption mechanism of the implanted noble gas and therefore we chose for thermal desorption measurements by stepwise heating. We are particularly interested in the activation energies and the amount of implanted noble gas. For each noble gas a series of ion doses is selected to determine the order of the desorption process and to obtain the collected noble gas atoms in the outer

\footnotetext{
* This work is part of the research program of the "Stichting voor Fundamenteel Onderzoek der Materie" (Foundation for Fundamental Research on Matter) and was made possible by financial support from the "Nederlandse Organisatie voor Zuiver Wetenschappelijk Onderzoek" (Netherlands Organization for the Advancement of Pure Research).
} 
layer as a function of the incident ion dose $N_{\mathrm{b}}$ at the surface. The collection curve for Ar is verified with RBS.

Also spectroscopic ellipsometric measurements are carried out during these bombardment and desorption experiments and are reported elsewhere [1]. For the interpretation of the desorption measurements of implanted noble gas ions in an amorphous silicon surface layer we present a simple model describing the desorption behaviour of implanted particles embedded in a surface layer. Therefore we shall first give a short review of a model of the kinetics of desorbed adsorbates, to see what they have in common.

\section{Kinetics of desorbed adsorbates}

Through heating of a surface, occupied by an adsorbed gas fraction $\theta_{0}=N_{\mathrm{o}} / \hat{N}$, a particle flux $J$ emerges due to desorption. For this particle flux the following relation is usually given in the literature [2-7]:

$J=-\hat{N} \mathrm{~d} \theta / \mathrm{d} t=\nu_{q} \hat{N} \theta^{q} \mathrm{e}^{-E_{\mathrm{s}} / k T}, \quad \theta(t), \theta(0)=\theta_{0}, \quad T(t), T(0)=T_{0}$,

in which $\hat{N}$ is the number of available sites at the surface per unit area, $E_{\mathrm{s}}$ the activation energy, $\theta$ the actual adsorbate fraction, $T$ the temperature, $k$ the Boltzmann constant, $q$ the order of the reaction and $\nu_{q}$ is called the rate constant. A typical value for $\nu_{1}$ is a value equal to the jump-frequency $\nu_{0}=10^{13} \mathrm{~s}^{-1}$. The probability term $\theta^{q}$ gives the mechanism of the desorption process. If the adsorbed particle $\left(\mathrm{A}_{\mathrm{s}}\right.$ ) leaves the surface without association then $q=1$ and the process is termed "first order". By associative desorption $\left(2 \mathrm{~A}_{\mathrm{s}} \rightarrow \mathrm{A}_{2}\right.$ (gas)) $q=2$, etc. The desorption rate is given by the term $\nu_{q} \exp \left(-E_{\mathrm{s}} / k T\right)$, assuming that the temperature dependence is of the Arrhenius form.

A widely used method is temperature programmed desorption, mostly with a linear temperature sweep of the form $T=T_{0}+\beta t$, where $\beta$ is the temperature rate. (Also $1 / T=1 / T_{0}-\alpha t$ is used with the advantage that eq. (1) integrates easily.) The maximum in the particle flux due to desorption follows from differentiation of eq. (1):

$$
\frac{\mathrm{d} J}{\mathrm{~d} t}=-\hat{N} \frac{\mathrm{d}^{2} \theta}{\mathrm{d} t^{2}}=\nu_{q} \hat{N} \theta^{q} \mathrm{e}^{-E_{\mathrm{s}} / k T}\left(\frac{q}{\theta} \frac{\mathrm{d} \theta}{\mathrm{d} t}+\frac{E_{\mathrm{s}}}{k T^{2}} \frac{\mathrm{d} T}{\mathrm{~d} t}\right)=0 .
$$

Substitution of eq. (1) and $\beta=\mathrm{d} T / \mathrm{d} t$ gives an implicit relation for the activation energy $E_{\mathrm{s}}$ :

$q \nu_{q} \theta_{\mathrm{m}}^{q-1}=\left(\beta E_{\mathrm{s}} / k T_{\mathrm{m}}^{2}\right) \mathrm{e}^{E_{\mathrm{s}} / k T_{\mathrm{m}}}$.

The maximum in the desorption flux at temperature $T_{\mathrm{m}}$ will depend on the value $\theta_{\mathrm{m}}=\theta\left(\theta_{0}, t_{\mathrm{m}}\right)$ if $q \neq 1$. For a second-order process $\theta_{\mathrm{m}} / \theta_{0} \approx 0.5[2]$. Eq. 
(3) shows that only for a first-order process the maximum in $J$ appears always at the same temperature $T_{\mathrm{m}}$ by varying $\theta_{0}$, at a constant $\beta$.

Differentiation of eq. (3) shows that for a second-order process the maximum in $J$ shifts to a lower $T_{\mathrm{m}}$ at increasing $\theta_{0}$ :

$\frac{\mathrm{d} T_{\mathrm{m}}}{T_{\mathrm{m}}}=(q-1) \frac{\mathrm{d} \theta_{\mathrm{m}}}{\theta_{\mathrm{m}}} \frac{-1}{2+E_{\mathrm{s}} / k T_{\mathrm{m}}}, \quad \frac{\mathrm{d} \theta_{\mathrm{m}}}{\theta_{\mathrm{m}}} \approx \frac{\mathrm{d} \theta_{0}}{\theta_{0}}$.

It is more usual to vary $\beta$ and, at a constant $\theta_{0}$, eq. (3) can then be written as:

$\ln \left(T_{\mathrm{m}}^{2} / \beta\right)=E_{\mathrm{s}} / k T_{\mathrm{m}}+\ln \left(E_{\mathrm{s}} / q v_{q} k \theta_{\mathrm{m}}^{q-1}\right)$.

The activation energy $E_{\mathrm{s}}$ follows from the graph of $\ln \left(T_{\mathrm{m}}^{2} / \beta\right)$ against $1 / T_{\mathrm{m}}$. The shape of the peaks can be used to get an indication of the order $q$ of the desorption mechanism.

Instead of this method it is also possible to arrive at a simple relation by using directly eq. (2):

$-q \frac{\mathrm{d} \theta}{\theta_{\mathrm{m}}}=\frac{E_{\mathrm{s}}}{k T_{\mathrm{m}}} \frac{\mathrm{d} T}{T_{\mathrm{m}}}$.

By using this relation, the temperature can be varied in an arbitrary monotonous way. Furthermore it is not necessary to know $\nu_{q}$ and $\theta_{\mathrm{m}}$ can be obtained from integrating $J$. However one must know $q$, therefore eq. (6) can only be used if the peaks do not shift with variation of $\theta_{0}$.

\section{Desorption behaviour of gas particles embedded in a surface layer}

\subsection{Monotonous heating}

The particle transport of noble gas atoms, with a density $n(x, y, z, t)$ in an amorphous surface layer $(0 \leqq z \leqq d)$ with thickness $d$ above a crystalline substrate $(z<0)$, can be described generally by means of a diffusion mechanism governed by the laws of Fick:

$\partial n / \partial t=-\operatorname{div} J=\operatorname{div}(D \operatorname{grad} n)$.

In general the calculation of the diffusion coefficient is a complex problem. However, if a model is adopted in which noble gas atoms diffuse due to a density gradient of defects, then the diffusion coefficient $D$ can be related to microscopic parameters [8]:

$D=D_{0} \mathrm{e}^{-E / k T}, \quad D_{0}=\gamma \nu_{0} a^{2} / l$.

In this relation (8) the parameters are the jump frequency $\nu_{0}$, the lattice constant $a$, the number of nearest possible sites $l$ and the probability $\gamma$ to find a vacancy on the neighbouring sites. 


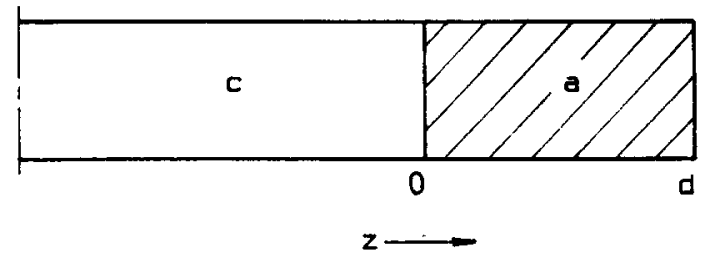

Fig. 1. Calculation of the desorption behaviour.

In the analysis of this section we will consider only one diffusion mechanism or that the desorption peaks due to different mechanisms are resolved. If the diffusion coefficient $D$ is only dependent on the temperature $T$ and if the particle density is only a function of $z, t$, then eq. (7) can be written as (see fig. 1)

$\partial n / \partial t=D_{0} \mathrm{e}^{-E / k T} \partial^{2} n / \partial z^{2}, \quad 0 \leqq z \leqq d$.

Firstly, we assume that the noble gas particles only diffuse in the amorphous layer to the interface amorphous silicon/vacuum at $z=d$ and cannot diffuse through the interface a-Si/c-Si at $z=0$. Secondly, it seems realistic to suppose that the noble gas particles leave the interface at $z=d$ directly, because the sojourn time of the noble gas atoms on surfaces above room temperature is very small [9]. We will also assume that no significant diffusion barrier occurs at the outermost atomic surface layers, for instance as due to surface reconstruction. As a starting condition we chose the implantation profile at $T=T_{0}$ and further the conditions at both interfaces at $z=0$ and $z=d$ :

$J(0, t)=-D \partial n(0, t) / \partial z=0$,

$n(d, t)=0$,

$n(z, 0)=$ implantation profile of noble gas at $T=T_{0}$.

With these conditions, eq. (9) can be solved by separation of variables: $n(z, t)=f(z) \theta(t)$. After substitution of such function eq. (9) can be formulated as follows:

$\frac{1}{\theta(t) D(T(t))} \frac{\mathrm{d} \theta}{\mathrm{d} t}=\frac{1}{f(z)} \frac{\mathrm{d}^{2} f}{\mathrm{~d} z^{2}}=-m^{2}$,

where $-m^{2}$ must be a constant. From the right-hand side it follows that $f=a \cos (m z)+b \sin (m z)$ and applying conditions (10) and (11) giving $b=0$, and for $m: m_{i}=(2 i+1) \pi / 2 d$. The relation for $\theta(t)$ looks like eq. (1) with $q=1$, so that it can be expected that this desorption process is of the first order:

$\mathrm{d} \theta / \mathrm{d} t=-m_{i}^{2} \theta(t) D(T(t)) \quad$ or $\quad \theta_{i}=c_{i} \exp \left[-m_{i}^{2} \int_{0}^{t} D\left(T\left(t^{\prime}\right)\right) \mathrm{d} t^{\prime}\right]$ 
As a more general solution having the above-mentioned conditions and with positive $i$ it follows:

$$
\begin{aligned}
n(z, t) & =\sum_{i=0}^{\infty} A_{i} \cos \left(m_{i} z\right) \exp \left[-m_{i}^{2} \int_{0}^{t} D(T) \mathrm{d} t^{\prime}\right] \\
J(d, t) & =-D \partial n(d, t) / \partial z \\
& =-D \sum_{i=0}^{\infty} A_{i}(-1)^{i} m_{i} \exp \left[-m_{i}^{2} \int_{0}^{t} D(T) \mathrm{d} t^{\prime}\right] .
\end{aligned}
$$

From eq. (12) the values $A_{i}$ can be calculated. Multiply relation (15a) by the orthogonal functions $\cos (2 j+1) \pi z / 2 d$ and take the integral from $z=0$ to $z=d$.

$A_{i}=\frac{2}{d} \int_{0}^{d} n(z, 0) \cos (2 i+1) \frac{\pi z}{2 d} \mathrm{~d} z, \quad i=0,1,2, \ldots$.

In general it will give $\left|A_{i}\right|<A_{0}$. Now if the value of

$$
\int_{0}^{t^{*}} \frac{\pi^{2} D\left(T\left(t^{\prime}\right)\right)}{4 d^{2}} \mathrm{~d} t^{\prime} \geqq 1 / 2
$$

then the first term of the series expansion is dominant and the other terms can be neglected due to the factor $(2 i+1)^{2}$ in the exponential. For $t>t^{*}$ the solution becomes:

$n(z, t)=A_{0} \cos (\pi z / 2 d) \theta(t), \mathrm{d} \theta / \mathrm{d} t=-\left(\pi^{2} D_{0} / 4 d^{2}\right) \theta \mathrm{e}^{-E / k T}$.

After introducing the mean particle density $\bar{n}$,

$\bar{n}(t)=\frac{1}{d} \int_{0}^{d} n(z, t) \mathrm{d} z$,

we obtain:

$\mathrm{d} \bar{n} / \mathrm{d} t=-J(d, t) / d=-\nu_{1} \bar{n} \mathrm{e}^{-E / k T}, \quad \nu_{1}=\pi^{2} D_{0} / 4 d^{2}, \quad t>t^{*}$.

Now we can estimate eq. (17) with $\mathrm{d} T=\beta \mathrm{d} t$ :

$$
\begin{aligned}
\int_{0}^{t} \frac{\pi^{2} D_{0} \mathrm{e}^{-E / k T}}{4 d^{2}} \mathrm{~d} t^{\prime} & =\frac{\nu_{1}}{\beta} \int_{T_{0}}^{T} \mathrm{e}^{-E / k T^{\prime}} \mathrm{d} T^{\prime} \\
& \approx \frac{\nu_{1}}{\beta}\left(\frac{k T^{2}}{E} \mathrm{e}^{-E / k T}-\frac{k T_{0}^{2}}{E} \mathrm{e}^{-E / k T_{0}}\right),
\end{aligned}
$$

where for the determination of the integral the first-order approximation is used as given by Lord and Kittelberger [4] and Redhead [2]. For a first-order process the right-hand term of eq. (21) is a monotonous function of $T$ and at $T=T_{\mathrm{m}}$ this term is exactly 1 , if we neglect the term containing $T_{0}$. This means that for a $T<T_{\mathrm{m}}$ condition (17) meets the requirement and the peak maximum can be considered as due to a first-order desorption process. 
Summarizing: it can be concluded that the desorption process of noble gas in a surface layer can be described, by using assumed boundary conditions, by first-order kinetics taking into account that the frequency factor $\nu_{1}$ is much lower than the jump frequency $\nu_{0}$ of eq. (8):

$\nu_{1} \approx \pi^{2} D_{0} / 4 d^{2}=\gamma\left(\nu_{0} / l\right)(\pi a / 2 d)^{2}, \quad \nu_{0} \approx 10^{13} \mathrm{~s}^{-1}$.

In the case of implantation of $2 \mathrm{keV} \mathrm{Ne}{ }^{+}$ions in a $\mathrm{Si}(111)$ crystal at normal incidence $a / d \approx 0.02$ and so $\nu_{1} \approx 10^{-3} \nu_{0}$ for $\gamma=1$. This conclusion is important, because Davies and Carter [10] assume at the interpretation of their implantation experiments a value $\nu_{1}$ equal to the jump frequency $\nu_{0}$ and then using eq. (3) with $q=1$ and the experimental value of $\beta$. However, if relation (6) is used, it is not necessary to know $\nu_{1}$.

We have to make another remark. Because we are now dealing with diffusion in a layer with thickness $d$, one should also observe peak shifts due to a varying $d$ or $\nu_{1}$. From relation (3), at a constant $\beta$ and $E$ with $q=1$ it follows for a varying $\nu_{1}$ :

$$
\frac{\delta \nu_{1}}{\nu_{1}}=-\left(2+\frac{E}{k T_{\mathrm{m}}}\right) \frac{\delta T_{\mathrm{m}}}{T_{\mathrm{m}}}=-2 \frac{\delta d}{d}+\frac{\delta \gamma}{\gamma}
$$

At increasing $d$ for instance, the peak shifts to a higher temperature.

\subsection{Stepwise heating}

From the point of view of the assumptions which have been made to arrive at eq. (20) a better method to determine the activation energy of the noble gas desorption mechanism is stepwise heating in stead of monotonous heating. In this case the only assumptions are that the diffusion coefficient $D$ will be independent of the concentration $n(x, y, z, t)$ and the gas will escape at the front of the sample and not at the back.

The gas stream $Q$, proportional to the particle flux $J$ from the sample, is measured with a mass spectrometer and can be given by:

$$
Q \propto \oiint_{\substack{\text { sample } \\ \text { surface }}} \boldsymbol{J} \cdot \mathrm{d} \boldsymbol{A}=-D(T) \oiint_{\substack{\text { sample } \\ \text { surface }}} \operatorname{grad} n \cdot \mathrm{d} \boldsymbol{A}=-\iiint_{\substack{\text { sample } \\ \text { volume }}} \frac{\partial n}{\partial t} \mathrm{~d} V .
$$

By increasing the temperature $T$ of the sample stepwise, in such way that at time $t_{i}^{-}, T=T_{i}$ and at time $t_{i}^{+}, T=T_{i+1}=T_{i}+\Delta T_{i}$ one will require for establishing a solution that:

$n\left(x, y, z, t_{i}^{-}\right)=n\left(x, y, z, t_{i}^{+}\right)$for $t_{i}^{+}-t_{i}^{-} \rightarrow 0$.

This means that $\operatorname{grad}(n)$ is also continuous at $t_{i}^{+}=t_{i}^{-}$but $\mathrm{d} \tilde{n} / \mathrm{d} t$ will change stepwise because $D(T)$ changes stepwise. We call the gas stream shortly before 
the temperature step $Q_{i}^{-}$and shortly after the step $Q_{i}^{+}$, then in accordance with eq. (8) and eq. (24) the following relation holds:

$$
\begin{aligned}
& \frac{Q_{i}^{+}}{Q_{i}^{-}}=\frac{D\left(T_{i+1}\right) \oiint \operatorname{grad} n \cdot \mathrm{d} \boldsymbol{A}}{D\left(T_{i}\right) \oiint \operatorname{grad} n \cdot \mathrm{d} \boldsymbol{A}}=\exp \left[-\frac{E}{k}\left(\frac{1}{T_{i+1}}-\frac{1}{T_{i}}\right)\right], \\
& \ln \frac{D_{I}}{D_{0}}=\sum_{i=0}^{I-1} \ln \frac{Q_{i}^{+}}{Q_{i}^{-}}=\sum_{i=0}^{I-1}-\frac{E}{k}\left(\frac{1}{T_{i+1}}-\frac{1}{T_{i}}\right)=-\frac{E}{k}\left(\frac{1}{T_{I}}-\frac{1}{T_{0}}\right) .
\end{aligned}
$$

The second term of eq. (26) which equals $\ln D_{I} / D_{0}$, can be determined experimentally for increasing $I$ and as a function of $1 / T_{I}$ it gives a slope $(-E / k)$.

\section{Experimental}

\subsection{Measurements}

The desorption experiments are carried out in a UHV system equipped with facilities for mass spectrometry, AES, mass-selective ion bombardment, sample temperature control and is described in more detail elsewhere [1]. The whole set-up is automated and controlled by an LSI-11 computer.

Before the noble gas ion implantation experiments started, the silicon (111) samples were cleaned by argon ion sputtering $\left(800 \mathrm{eV}, 2 \times 10^{-6} \mathrm{~A} \mathrm{~cm}^{-2}\right.$ and at $45^{\circ}$ to the surface normal) followed by an anneal step at $1100 \mathrm{~K}$ during $1 \mathrm{~h}$ and afterwards checked by AES.

The bombardments were carried out using a $2.2 \mathrm{kV}$ mass-selective ion beam of $\mathrm{Ne}^{+}, \mathrm{Ar}^{+}$or $\mathrm{Kr}^{+}$at normal incidence with a beam current of $115 \mathrm{nA} \mathrm{cm}{ }^{-2}$. For each noble gas mass, a series of about 7 characteristic ion doses are chosen, firstly to obtain collection curves of implanted atoms as a function of striking dose $N_{\mathrm{b}}$ and secondly to determine the order of the desorption mechanism.

After implantation of the $\mathrm{Si}(111)$ surfaces with noble gas ions at room temperature $T_{0} \approx 300 \mathrm{~K}$, the temperature is raised in 50 steps up to $1050 \mathrm{~K}$. The same temperature increase is always applied and realized by introducing a stepwise increasing current through the sample, controlled by an LSI. Because, as it appears, there is almost no gas release below $500 \mathrm{~K}$ and also because the ellipsometric values change slowly below $500 \mathrm{~K}$, we have taken a variable heating rate: faster at the beginning, becoming slower towards the end, where the interesting features occur. The measuring cycle with the datapoint sampling during a desorption experiment is given in fig. 2 .

The partial pressure $p$ is measured with a quadrupole (Riber QMM16) 


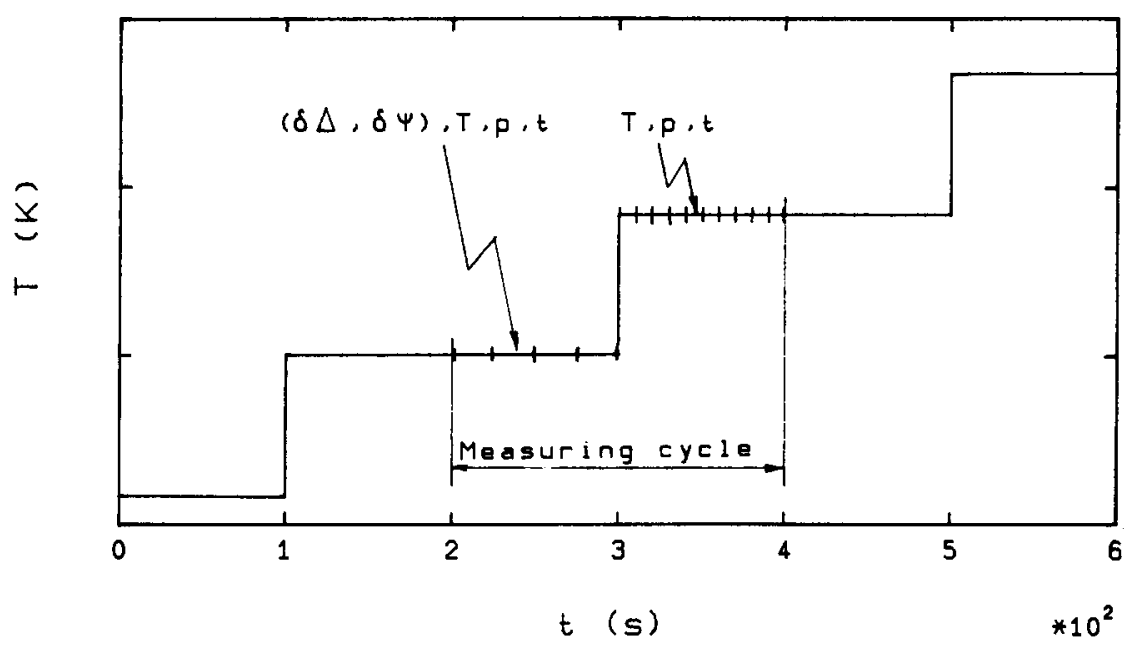

Fig. 2. The measuring cycle during desorption experiments.

adjusted to the singular ionized noble gas atoms. This measuring method makes it possible to determine activation energies in two ways and to measure, at the same temperatures, the ellipsometric parameters $\Delta$ and $\psi$ at four different photon energies. These optical measurements are reported elsewhere [1].

\subsection{Calibration}

Before starting the desorption measurements the quadrupole has been calibrated by means of a pump speed measurement. In fig. 3 the gas streams $Q$

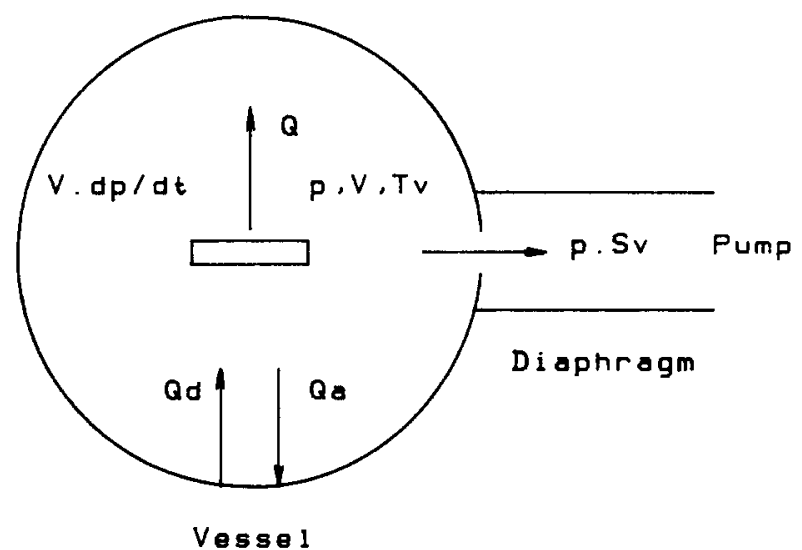

Fig. 3. Schematic representation of the gas streams $Q$ in the UHV system during desorption experiments. 
Table 1

Time constant $\tau$ of the noble gas compared with the theoretical expression $\tau \propto \sqrt{m}$

\begin{tabular}{llll}
\hline Noble gas & $\tau(\mathrm{s})$ & $m(\mathrm{amu})$ & $\tau / \sqrt{m}$ \\
\hline $\mathrm{Ne}$ & 42.4 & 20 & 9.48 \\
$\mathrm{Ar}$ & 62.5 & 40 & 9.88 \\
$\mathrm{Kr}$ & 88.1 & 84 & 9.61 \\
\hline
\end{tabular}

in the UHV system are shown schematically during the desorption measurements. Here the adsorption gas stream $Q_{\mathrm{a}}$ is a function of the partial pressure $p$ inside the system and the wall temperature $T_{\mathrm{v}}, Q_{\mathrm{a}}=p f_{\mathrm{a}}\left(T_{\mathrm{v}}\right)[11,12]$ and the desorption gas stream $Q_{\mathrm{d}}$ from the wall is only a function of the wall temperature, $Q_{\mathrm{d}}=f_{\mathrm{d}}\left(T_{\mathrm{v}}\right)[11,12]$. The pump speed through the diaphragm is called $S_{\mathrm{v}}$. The relation between the gas stream $Q$ from the sample and flux $J$ is given by:

$Q=-k T_{\mathrm{v}} \frac{\mathrm{d}}{\mathrm{d} t} \iiint_{\substack{\text { sample } \\ \text { volume }}} n \mathrm{~d} V=k T_{\mathrm{v}} \oiint_{\substack{\text { sample } \\ \text { surface }}} J \cdot \mathrm{d} \boldsymbol{A}$.

The balance equation for the noble gas is:

$Q+Q_{\mathrm{d}}-\left[f_{\mathrm{a}}\left(T_{\mathrm{v}}\right)+S_{\mathrm{v}}\right] p=Q+Q_{\mathrm{d}}-S p=V \mathrm{~d} p / \mathrm{d} t$,

where we have introduced a total pump speed $S$. Before the desorption measurement starts, $Q=0$ and the final partial pressure becomes $p_{\infty}=Q_{\mathrm{d}} / S$. Defining $\Delta p=p-p_{\infty}$ so that:

$Q / S=\Delta p+\tau \mathrm{d} \Delta p / \mathrm{d} t, \quad \tau=V / S$.

By means of a pump speed measurement the time constant $\tau$ and the final partial pressure $p_{\infty}$ are determined.

The pump speed $S_{\mathrm{v}}$ is given by $1 / S_{\mathrm{v}}=1 / S_{\mathrm{T}}+R_{\mathrm{L}}+R_{\mathrm{D}} \approx R_{\mathrm{L}}+R_{\mathrm{D}}$ with $S_{\mathrm{T}}$ the pump speed of the turbomolecular pump, $R_{\mathrm{L}}$ the pump resistance of the pumpline and $R_{\mathrm{D}}$ the resistance of the diaphragm. Both $R_{\mathrm{L}}$ and $R_{\mathrm{D}}$ are proportional to the root of the mass $m$ of the noble gas atom [12] and thus $\tau \propto \sqrt{m}$, see table 1 .

The quadrupole produces a voltage $U$ proportional to the partial pressure $p=a U+b$, so $\Delta p=p-p_{\infty}=a\left(U-U_{\infty}\right)$ and is fully determined without the constant $a$. Therefore we introduce a reference pressure $p_{\text {ref }}=a U_{\text {ref }}$.

\subsection{Methods of calculation}

In figs. 4-6 the results for $Q / Q_{\text {ref }}$ and $N_{\mathrm{d}} / N_{\text {ref }}$ are given for $\mathrm{Ne}, \mathrm{Ar}$ and $\mathrm{Kr}$. By means of fig. 7 the calculations will be explained. This desorption experiment is part of the $\mathrm{Kr}-1$ experiment as given in fig. $6\left(N_{\mathrm{b}}=0.0475\right.$ 

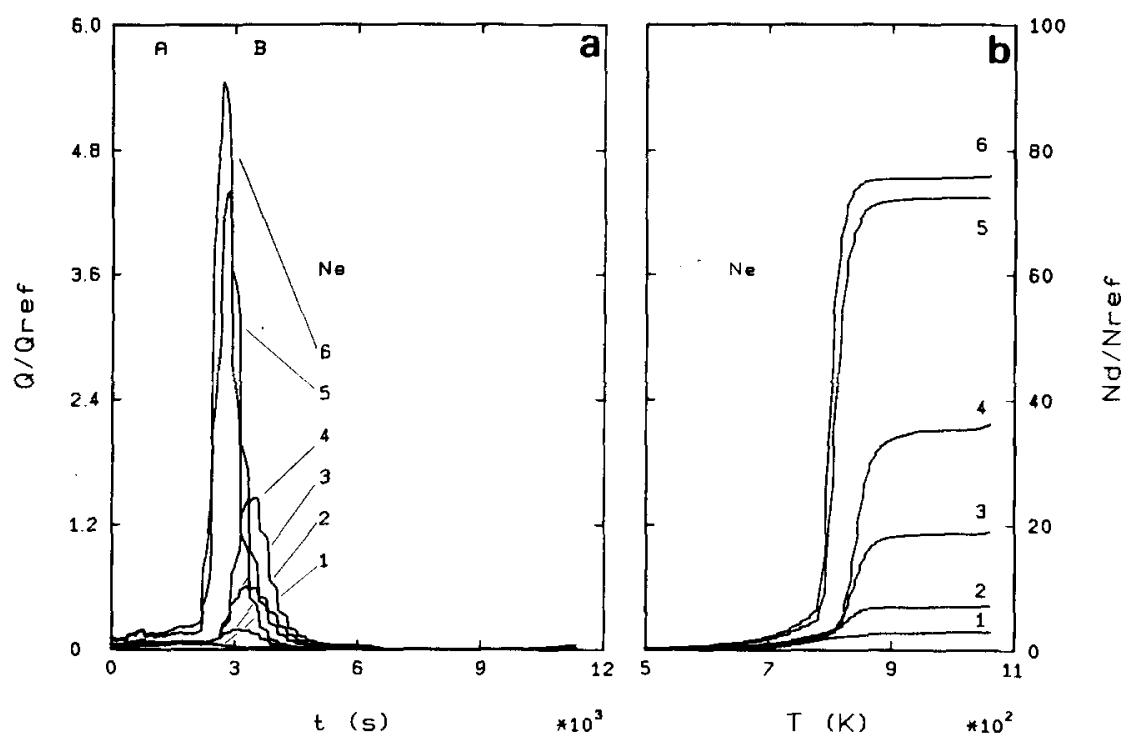

Fig. 4. The desorption curves of $\mathrm{Ne}$ (a) and the amount of desorbed noble gas as a function of temperature (b). The numbers 1-6 denote different ion-bombardment doses. The letters $\mathrm{A}$ and $\mathrm{B}$ correspond to different desorption mechanisms.

$\AA^{-2}$ ). For the calculation of $Q(t)$, eq. (29) has been scaled with a gauge factor $p_{\text {ref }}\left(p_{\text {ref }}=f(\right.$ noble gas $\left.)\right)$ :

$\frac{Q}{Q_{\text {ref }}}=\frac{\Delta p}{p_{\text {ref }}}+\tau \frac{\mathrm{d}\left(\Delta p / p_{\text {ref }}\right)}{\mathrm{d} t}, \quad Q_{\text {ref }}=S p_{\text {ref }}$.

For figs. $4 \mathrm{a}, 5 \mathrm{a}$ and $6 \mathrm{a}$ the derivatives are calculated from three successive measuring points $\Delta p(t) / p_{\text {ref }}$, except at a temperature step. At these steps only the differential quotients at both sides of the step are used. After that, the data points $Q / Q_{\text {ref }}$ are smoothed. These figures serve only for a mutual comparison in relation to the peak position and/or peak shift. The same temperature increase has always been used.

The desorption dose $N_{\mathrm{d}}$ can be calculated at every time from the time integral of eq. (30):

$A N_{\mathrm{d}}(t)=\int_{0}^{t} \frac{Q\left(t^{\prime}\right)}{k T_{\mathrm{v}}} \mathrm{d} t^{\prime}=A N_{\text {ref }}\left[\frac{\Delta p(t)}{p_{\text {ref }}}+\int_{0}^{t} \frac{\Delta p\left(t^{\prime}\right)}{p_{\text {ref }}} \frac{\mathrm{d} t^{\prime}}{\tau}\right]$,

with scaling factor $A N_{\text {ref }}=\tau Q_{\text {ref }} / k T_{\mathrm{v}}=V p_{\text {ref }} / k T_{\mathrm{v}}$. The implantation area $A$ is about $1 \mathrm{~cm}^{2}$. For a noble gas always the same beam profile has been used. The integral in the right term of eq. (31) can be calculated by means of either the Simpson rule or the trapeze rule at the temperature steps. If there is only one 

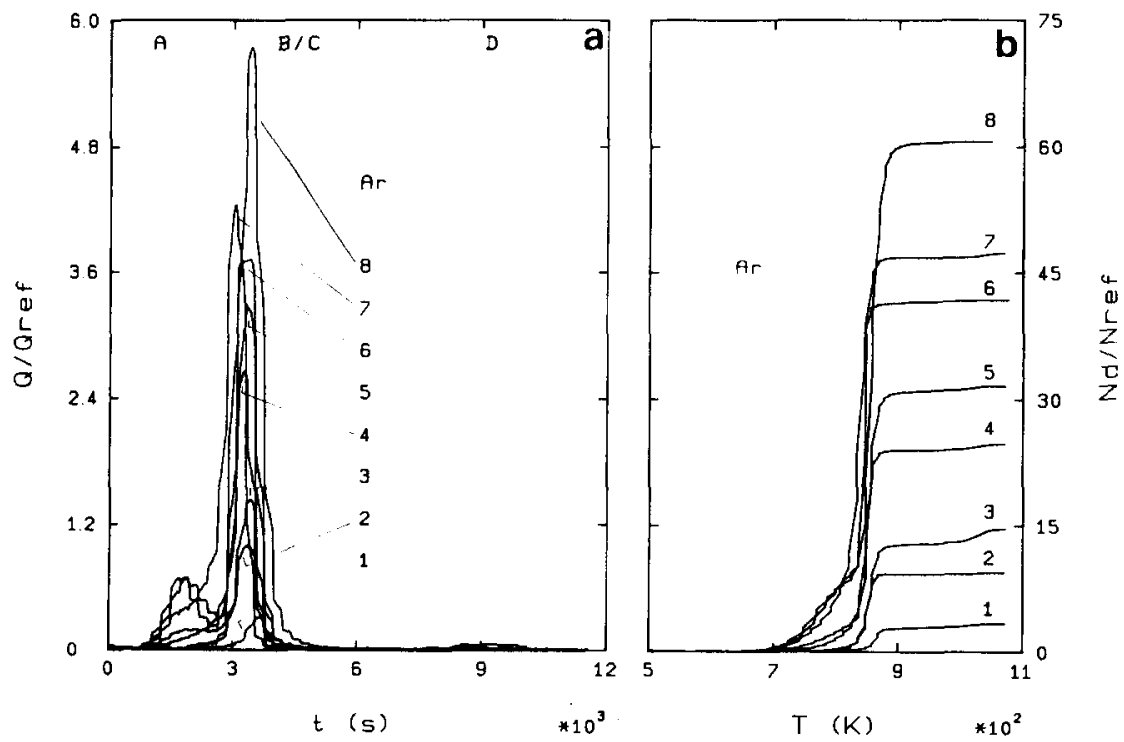

Fig. 5. The desorption curves (a) and desorbed doses (b) for Ar. The numbers 1-8 correspond with ion-bombardment doses. The letters A-D correspond to different desorption mechanisms.
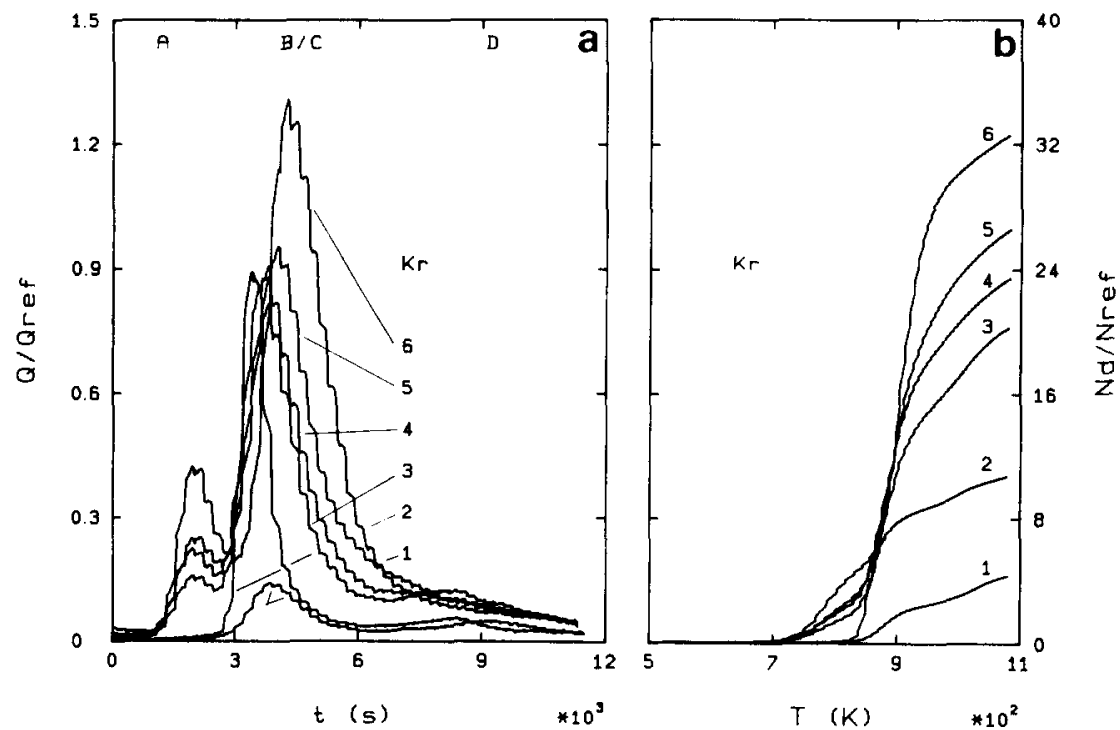

Fig. 6. The desorption curves (a) and desorbed doses (b) for $\mathrm{Kr}$. The numbers 1-6 denote different ion-bombardment doses and the letters A-D correspond to different desorption mechanisms. Figs. 4, 5 and 6 can be compared. 
desorption peak, the value $N_{\mathrm{p}}=\hat{N}_{\mathrm{d}}-N_{\mathrm{d}}$ gives the actual number of noble gas atoms present in the sample, which will have the sample by the same desorption mechanism.

\subsubsection{Monotonous heating}

First the relations from section 2 are applied on the basis of figs. $7 \mathrm{a}-7 \mathrm{c}$. By taking up the stepwise temperature increase as a monotonous increase, a temperature peak $T_{\mathrm{m}}=880 \mathrm{~K}$ at time $t_{\mathrm{m}} \approx 4000 \mathrm{~s}$ has been found from fig. 6 , or fig. $7 \mathrm{~d}$. From fig. $7 \mathrm{a}$ it follows for the temperature increase $\bar{\beta}=\overline{\mathrm{d}} \overline{\mathrm{T} / \mathrm{d} t}=$ $0.042 \mathrm{~K} / \mathrm{s}$ and from fig. $7 \mathrm{c}$ follows $\left(1 / N_{\mathrm{p}}\right) \mathrm{d} N_{\mathrm{p}} / \mathrm{d} t=-1.6 \times 10^{-3} \mathrm{~s}^{-1}$ at $t=t_{\mathrm{m}}$, with the result that by means of eq. (6) $E=2.5 \mathrm{eV}$. The accuracy of this result is especially determined through the value of $N_{\mathrm{p}}$ at $t=t_{\mathrm{m}}$, where $N_{\mathrm{p}}$ corresponds to the amount of particles still present but will leave the sample by the same mechanism. Therefore we have used the value $N_{\mathrm{p}} / N_{\text {ref }}=0.80$ from section 4.3.2. If there is only one peak present, the application of eq. (6) will be straightforward.

\subsubsection{Stepwise heating}

The gas streams $Q^{+}, Q^{-}$from eq. (26) are determined by describing the pressure course, before and after the temperature step, as $\Delta p_{i}^{+}(t) / p_{\text {ref }}=$ $\Delta p\left(t_{i}\right) / p_{\text {ref }}+\alpha^{+}\left(t-t_{i}\right)+\beta^{+}\left(t-t_{i}\right)^{2}+\ldots$ for $Q_{i}^{+}$and corresponding for $Q_{i}^{-}$. The coefficients $\alpha^{ \pm}$and $\beta^{ \pm}$can be calculated by means of linear regression. Applying eq. (30) gives $Q^{ \pm}, Q_{i}^{+} / Q_{\text {ref }}=\Delta p\left(t_{i}\right) / p_{\text {ref }}+\tau \alpha^{+}$. For the linear regression we take only data points for $\Delta p_{i}^{+}$as long as $\Delta p$ increases after a temperature step or for a $\Delta t=t-t_{i}<\tau$. Neglecting the second-order terms can give errors of about $5 \%$ in the value of $Q^{+} / Q^{-}$. The value of the second-order term is about $\Delta t / 2 \tau$ times the first-order term, where $\Delta t$ corresponds to the time interval for linear regression. (It should be noted to choose $\Delta t \ll \tau$.)

In fig. $7 \mathrm{~d}$ the values of $Q^{ \pm}$are given and connected by perpendicular lines. Relation (26) is shown in fig. 7e. The value $D_{\text {ref }}$ presents a value of $D$ at a reference point, the start of the calculation. It follows from the slope that $E / k=2.73 \times 10^{4} \mathrm{~K}$ respectively $E=2.35 \mathrm{eV}$. The time interval $2500<t<$ $5000 \mathrm{~s}$ in figs. $7 \mathrm{a}-7 \mathrm{~d}$ corresponds with $1.05 \times 10^{-3}<1 / T<1.25 \times 10^{-3} \mathrm{~K}^{-1}$, so even for $t<2500 \mathrm{~s}$ the desorption mechanism can be described by $E=2.35$ $\mathrm{eV}$, and the activation energy is determined from values for $T<900 \mathrm{~K}$.

Now the activation energy $E$ is known, eq. (1) can be rewritten:

$$
\frac{Q}{Q_{\text {ref }}} \mathrm{e}^{E / k T}=\nu_{q} \tau \frac{\hat{N}}{N_{\text {ref }}}\left(\frac{\hat{N} / N_{\text {ref }}-N_{\mathrm{d}} / N_{\text {ref }}}{N / N_{\text {ref }}}\right)^{q} \text {. }
$$

For $t>t^{*}$, see section 3.1, assuming the desorption kinetics can be described with a first-order process, this means that only for $q=1$ the graph $(Q /$ $\left.Q_{\text {ref }}\right) \exp (E / k T)$ against $N_{\mathrm{d}} / N_{\text {ref }}$ gives a straight line with a slope $-\nu_{1} \tau$. Eq. 


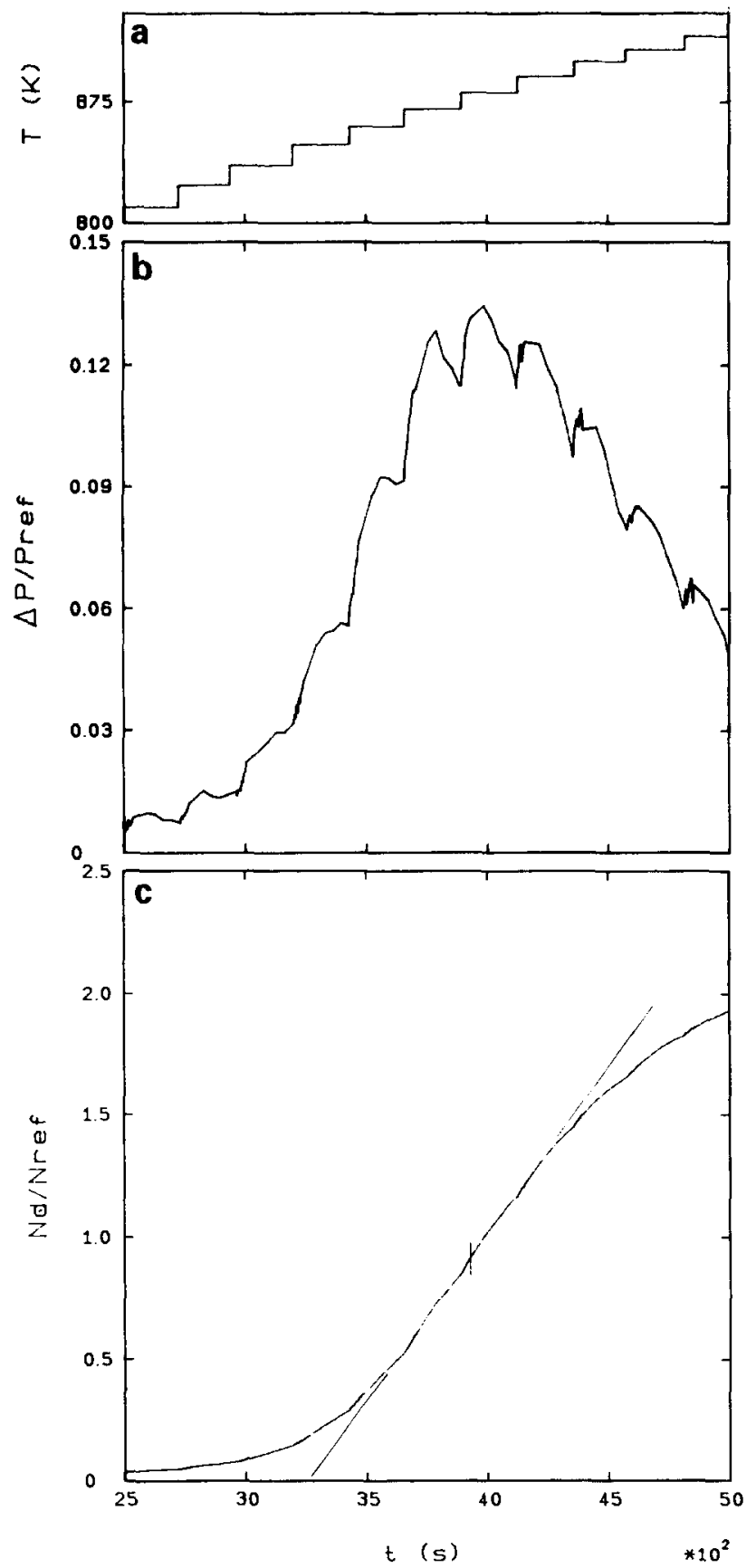

Fig. 7. (a) Part of the applied temperature increase. (b) Part of the relative pressure increase for the $\mathrm{Kr}-1$ case at peak B. (c) Part of the amount of desorbed noble gas for the $\mathrm{Kr}-1$ case at peak $\mathrm{B}$, see fig. 6 number 1 , letter B. (d) The calculated gas streams $Q^{+}$and $Q^{-}$, connected with perpendicular lines. The values are calculated from the results in fig. $7 \mathrm{~b}$. (e) The calculated diffusion coefficient at peak B of the Kr-1 case. (f) Verification of a first-order process in the case of peak B of the $\mathrm{Kr}-1$ case. 

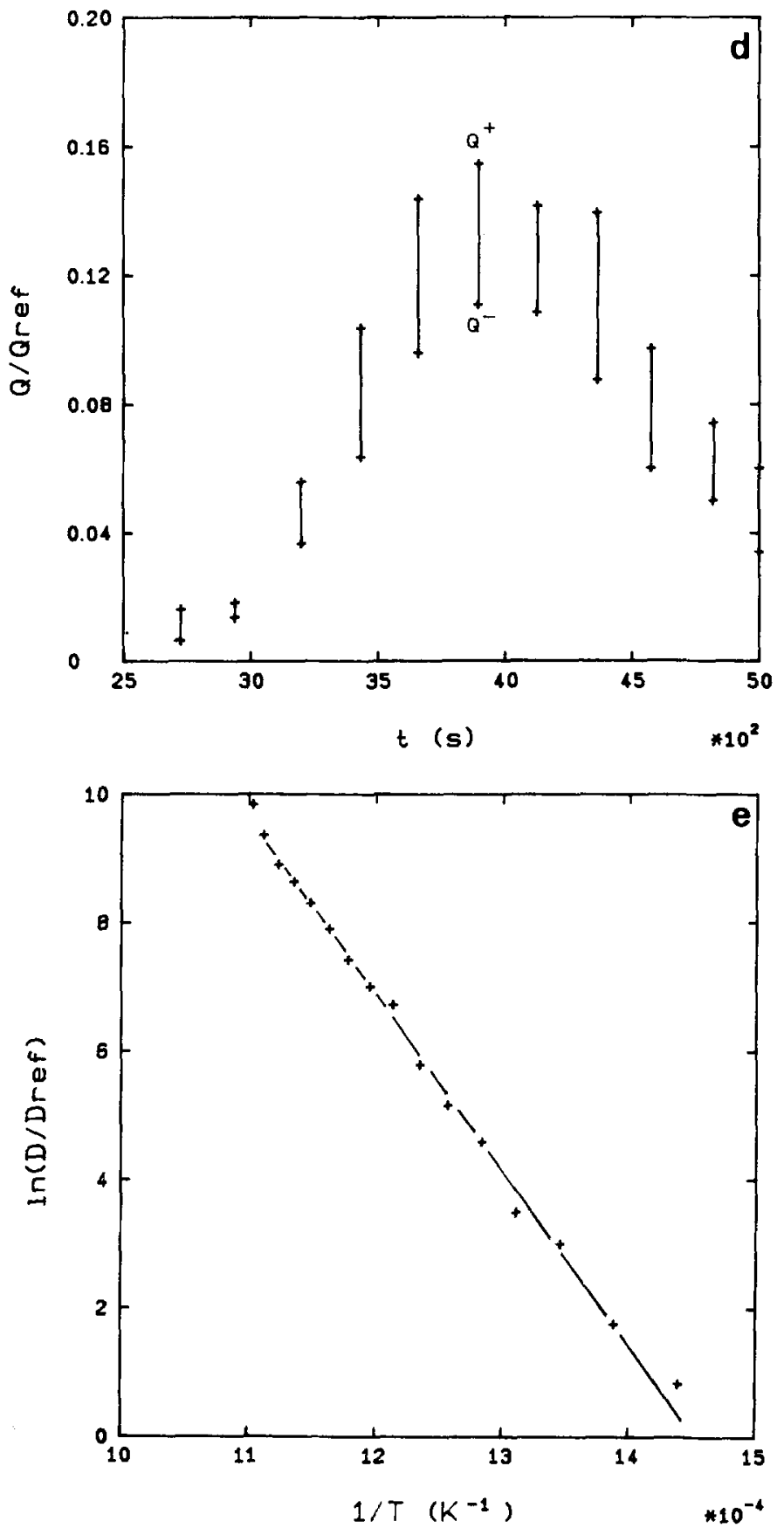

Fig. 7. Continued. 


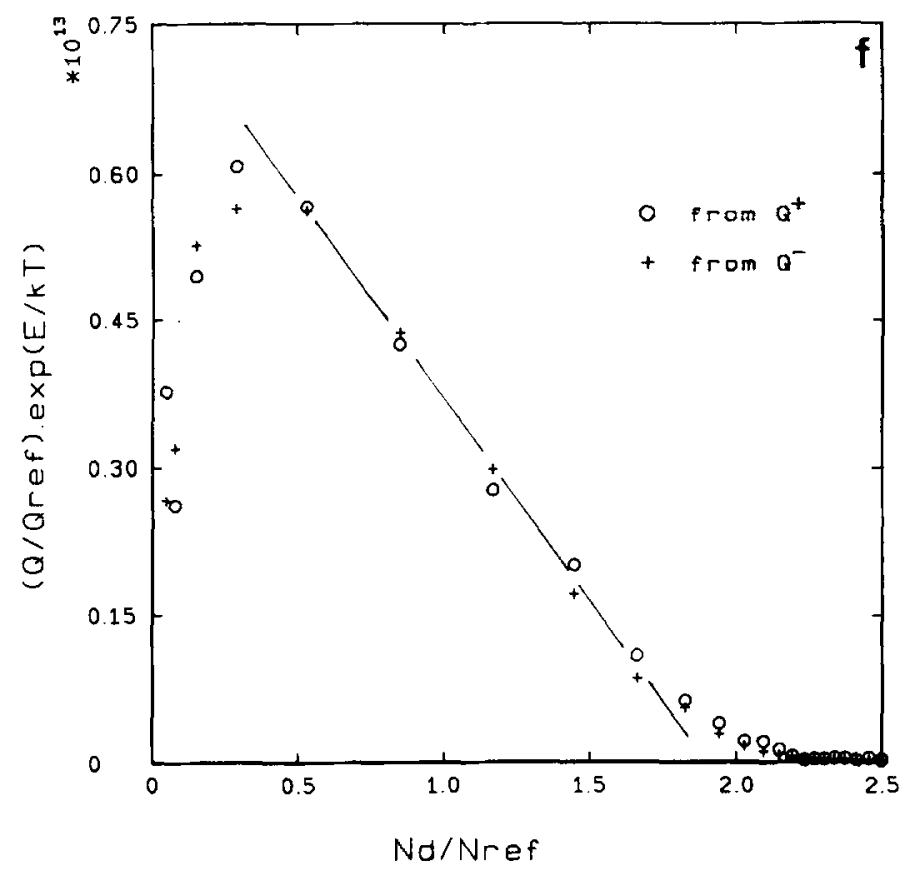

Fig. 7. Continued.

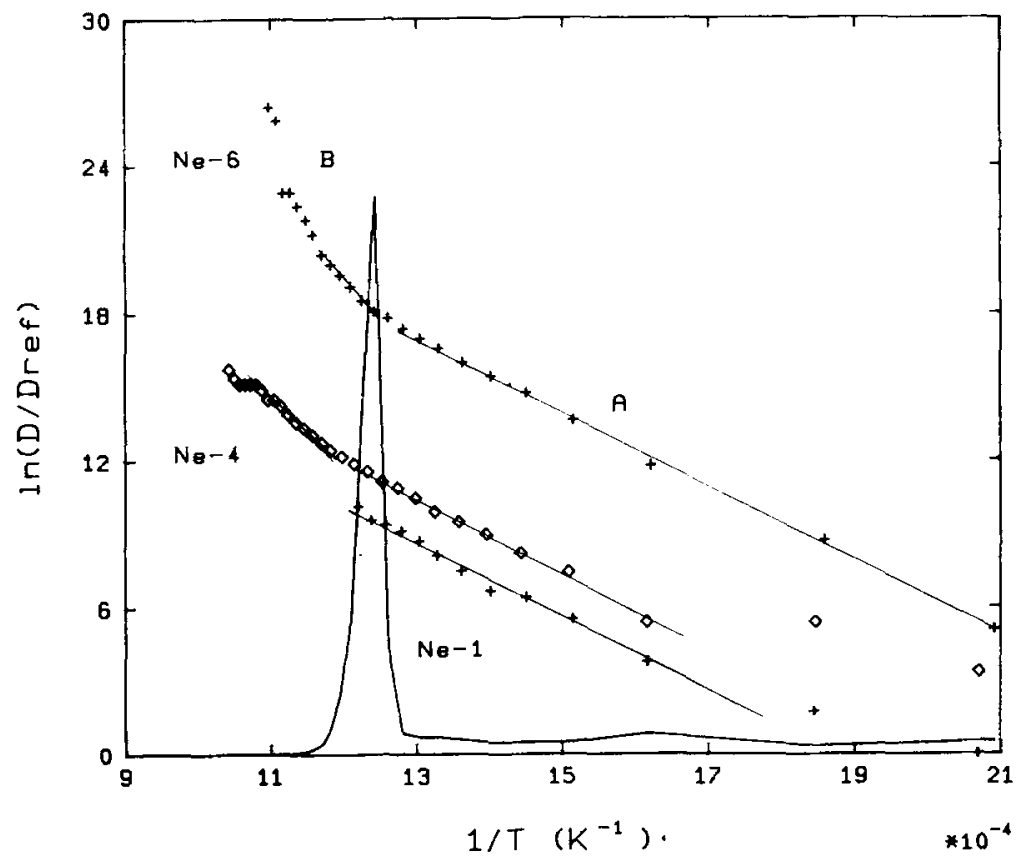

Fig. 8. Results for Ne. The letters and numbers correspond to fig. 4. The desorption curve Ne-6 is also included as a reference. 


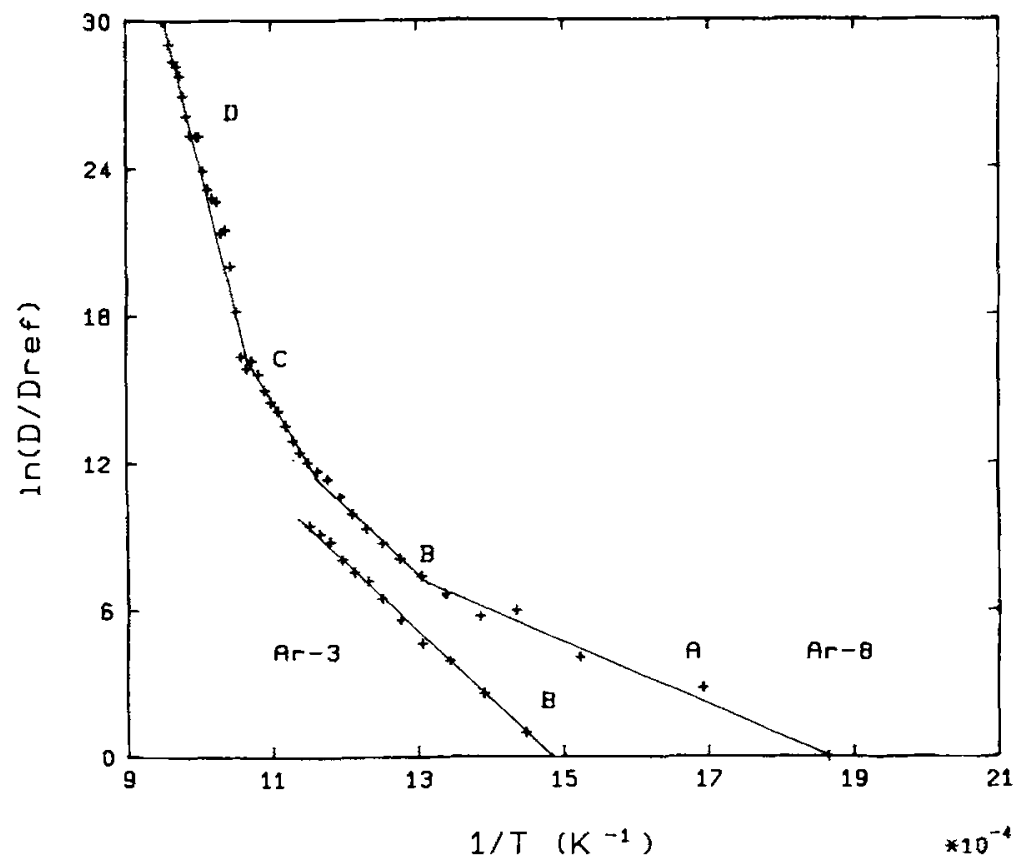

Fig. 9. Results for Ar. The letters and numbers correspond to fig. 5.

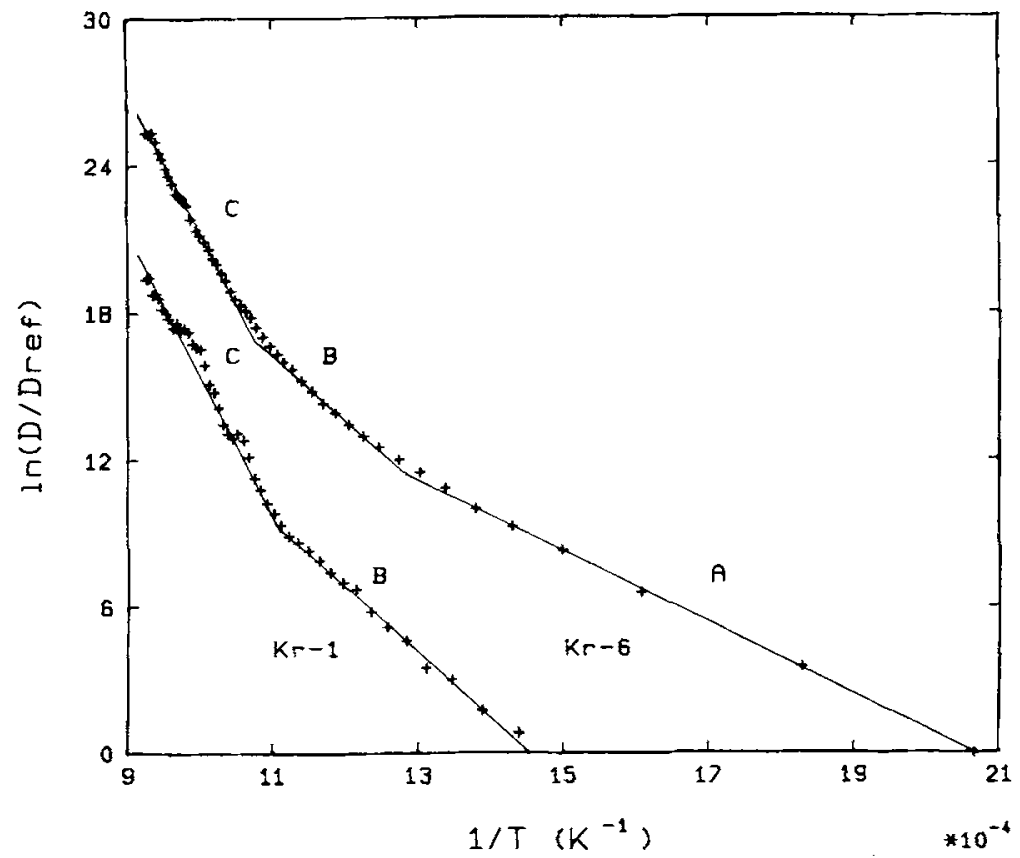

Fig. 10. Results for $\mathrm{Kr}$. The letters and numbers correspond to figs. 6 and 7. Figs. 8,9 and $10 \mathrm{can}$ be compared. 
(32) is shown in fig. $7 \mathrm{f}$ and a linear relation is found for $0.5<N_{\mathrm{d}} / N_{\text {ref }}<1.7$. The intersection of the slope with the axis gives $\hat{N} / N_{\text {ref }}=1.85$, while at $N_{\mathrm{d}} / N_{\text {ref }}=1.05$ the desorption peak occurs. Finally it follows from the slope that $\nu_{1}=4 \times 10^{10} \mathrm{~s}^{-1}$. The value of $\hat{N}$ and $\nu_{1}$ are very sensitive for a small error in $E$.

\subsection{Desorption spectra}

All desorption spectra from figs. $4 \mathrm{a}, 5 \mathrm{a}$ and $6 \mathrm{a}$ show at $t \approx 2000 \mathrm{~s}\left(T_{\mathrm{m}}=775\right.$ $\mathrm{K}$ ) a desorption peak with the peak maximum localized at the same place at increasing bombardment dose. This peak, called A, represents a first-order desorption process.

Further, in all the spectra a principal peak is present and shifts with an increasing dose. These peaks correspond to two mechanisms, called $\mathrm{B} / \mathrm{C}$. The spectra for $\mathrm{Ar}$ and $\mathrm{Kr}$ show a third D-peak. The graphs 8-10 have been obtained by using the calculation method of section 4.3.2.

First we analyse the results for $\mathrm{Kr}$. The complete results for the example $\mathrm{Kr}-1$ from section 4.3 are given in fig. 10. For $\mathrm{Kr}-1$ two activation energies can be distinguished, $E_{\mathrm{B}}=2.35 \mathrm{eV}$ and $E_{\mathrm{C}}=4.4 \mathrm{eV}$. The value of $\mathrm{E}_{\mathrm{C}}$ is determined for $1.05 \times 10^{-3}<1 / T<1.14 \times 10^{-3} \mathrm{~K}^{-1}$, because for $1 / T<1.05$ $\times 10^{-3} \mathrm{~K}^{-1}$ the peak $\mathrm{D}$ becomes visible. At increasing bombardment dose, desorption peak $\mathrm{A}$ arises, as shown in fig. 6a with an activation energy $E_{\mathrm{A}}=1.25 \mathrm{eV}$, and the slopes in fig. 10 for peak B and C stay the same.

Exactly the same remarks can be made for the argon spectra, see figs. 5a and 9 , with about the same numerical values. However, the principal desorption peak $\mathrm{B} / \mathrm{C}$ is smaller, which can be explained by a lower peak concentration of $\mathrm{C}$.

In the Ne-spectra the desorption mechanism $\mathrm{C}$ is absent, or is so small that it cannot be detected, while at low ion dose peak $\mathrm{A}$ is already present in comparison with the argon and krypton spectra. For $\mathrm{Ar}$ and $\mathrm{Kr}$ the peak $\mathrm{A}$ appears at a higher ion dose after peak B/C has become apparent. Only in the Ar spectra of fig. 9 can the activation energy for mechanism $D$ be qualitatively distinguished with a value of $E_{\mathrm{D}} \approx 10 \mathrm{eV}$.

Table 2

Activation energies $(E)$ and rate constants $\left(\nu_{1}\right)$ of noble gases

\begin{tabular}{|c|c|c|c|c|c|c|c|c|c|}
\hline \multirow{3}{*}{$\begin{array}{l}\text { Noble } \\
\text { gas }\end{array}$} & \multirow{2}{*}{\multicolumn{3}{|c|}{$\frac{\text { Monotonous heating }}{E(\mathrm{eV})}$}} & \multicolumn{6}{|c|}{ Stepwise heating } \\
\hline & & & & \multicolumn{4}{|l|}{$E(\mathrm{eV})$} & \multicolumn{2}{|l|}{$\nu_{1}\left(\mathrm{~s}^{-1}\right)$} \\
\hline & A & B & $\mathrm{C}$ & A & B & C & D & A & B \\
\hline $\mathrm{Ne}$ & $\sim 1.2$ & 2.6 & - & $\sim 1.25$ & 2.5 & - & - & $2 \times 10^{5}$ & $4 \times 10^{11}$ \\
\hline Ar & 1.3 & - & 4.2 & 1.20 & 2.4 & 4.5 & $\sim 10$ & $2 \times 10^{5}$ & $9 \times 10^{10}$ \\
\hline $\mathrm{Kr}$ & 1.2 & 2.5 & - & 1.23 & 2.35 & 4.4 & - & $2 \times 10^{5}$ & $4 \times 10^{10}$ \\
\hline
\end{tabular}


Summarizing, it can be concluded that at the diffusion during the desorption process at least four mechanisms play a role. The influence of the type of noble gas arises in the rate constant $\nu_{1}$ and/or the relative peak contributions. We are not able to determine the rate constant $\nu_{\mathrm{C}}$ because it is not a first-order diffusion mechanism. The results are listed in table 2.

\subsection{Collection curves}

At $T=1075 \mathrm{~K}$ the final values $\hat{N}_{\mathrm{d}}$ from figs. $4 \mathrm{~b}, 5 \mathrm{~b}$ and $6 \mathrm{~b}$ correspond with the dose in the amorphous surface layer after a bombardment dose $N_{\mathrm{b}}$ on the surface of the sample. For $\mathrm{Kr}$ there is still a release of a small amount of gas at $1100 \mathrm{~K}$. In order to obtain the characteristic dose $N_{\mathrm{b}}$ where saturation occurs, we have chosen $N_{\text {ref }}$ in such way that for low dose $\hat{N}_{\mathrm{d}}=N_{\mathrm{b}}$, see fig. 11 . Because assuming a low constant reflection coefficient $r$ at the surface, the collected dose will initially be $(1-r) \hat{N}_{\mathrm{b}}$. At $2.2 \mathrm{keV}$ the calculated [13] reflection coefficient for $\mathrm{Ne}$ is 0.04 and for Ar and $\mathrm{Kr}$ about 0 . At a large dose a saturation will arise which can be described by means of the implantation model for the steady state of Schulz and Wittmaack [13].

The purpose of the RBS measurements is to check the shape of the collection curve and to obtain implantation profiles of the noble gas. The beam profile used for implantations shows a lower current density at the outside of the cylindrical beam. The surface of the target which is hit by the outside of the beam will therefore reach the steady state after a higher ion

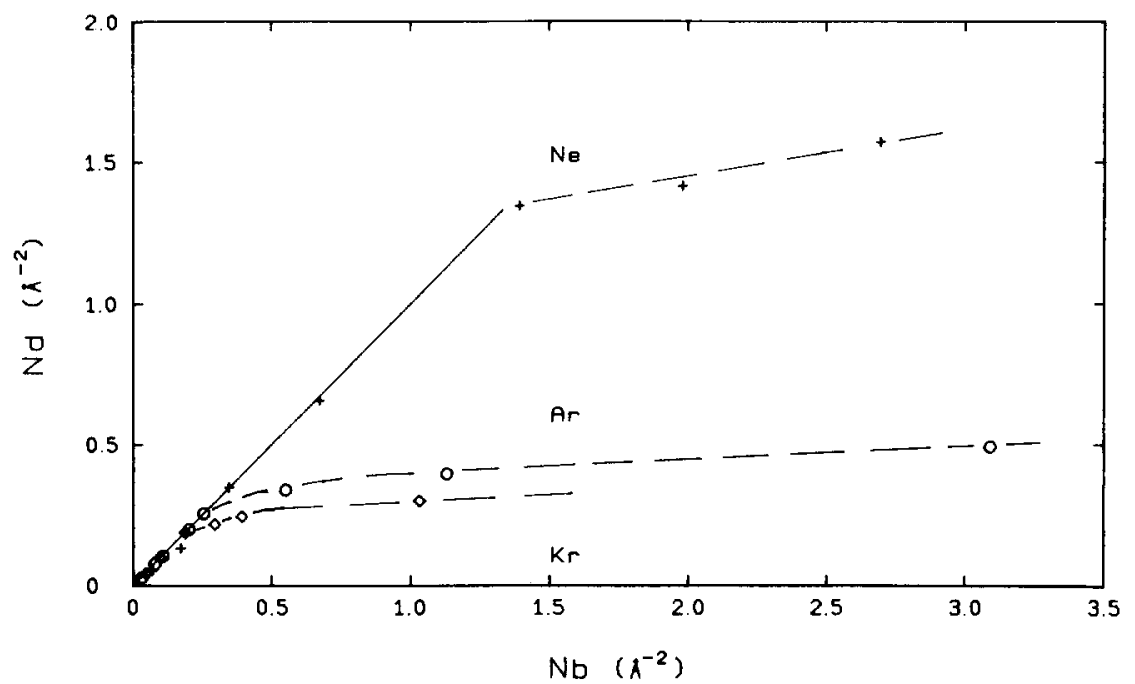

Fig. 11. The total desorbed dose $N_{\mathrm{d}}$ as a function of impinging ion dose $N_{\mathrm{h}}$. These graphs are called the collection curves. 
dose. Such an effect can explain the increase of the number of collected atoms, after a steady state at the centre of the target is reached, see fig. 11. Because RBS resolved elements with an atomic mass higher than the substrate atoms well, it is obvious to check the $\mathrm{Ar}$ or $\mathrm{Kr}$ results.

Three samples were implanted with $\mathrm{Ar}^{+}$(normal incidence, $2.2 \mathrm{kV}$ ) with suitably chosen doses $\left(0.090,0.36,1.44 \AA^{-2}\right)$. So we used two doses before the bend in fig. 11, and one dose after this bend. Six days after the implantations the RBS measurements were carried out. From the values obtained for $\nu_{1}$ and $E$ it is easy to check that the amount of Ar which leaves the sample in six days due to the observed desorption mechanisms at room temperature can be neglected.

The results of the RBS measurements, using a mono-energetic $\mathrm{He}^{+}$beam of $2 \mathrm{MeV}$ at normal incidence and random direction with a beam current of 10-30 nA, a beam area of $\sim 1 \mathrm{~mm}^{2}$ and a silicon surface-barrier detector (Ortex) at an scattering angle of $170^{\circ}$ are shown in fig. 12. In this figure the total backscattered yield due to the Ar peak, measured at the centre of the implanted area, is compared with the measured desorbed doses from fig. 11. Regarding the accuracy of the measurements, we observe a straight line. Therefore the influence of the beam profile used on the shape of the collection

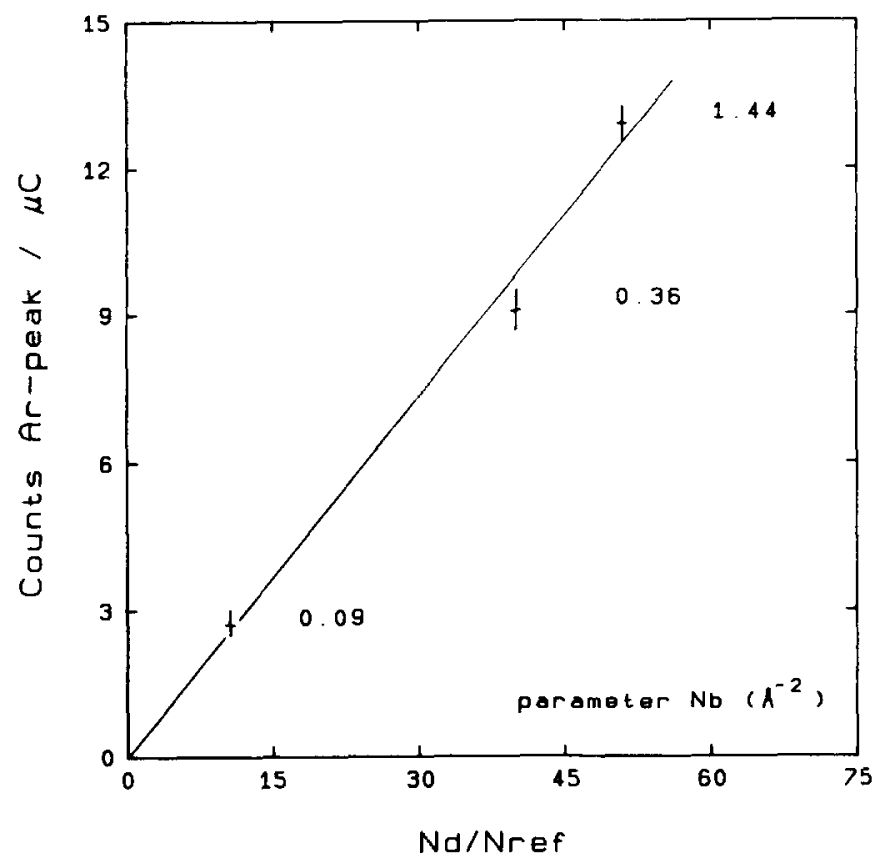

Fig. 12. Verification of the collection curve of Ar with RBS measurements, for three bombardment doses $N_{\mathrm{b}}$. 
curve can be neglected. Furthermore from the level of the Si shoulder obtained in random direction one can establish an absolute value of the implanted $\mathrm{Ar}$ dose, which is $0.85 \pm 0.10$ of the striking dose in the region where $N_{\mathrm{d}}$ is proportional to $N_{\mathrm{b}}$. This value can be explained by a low reflection coefficient of the ions implanted at $2.2 \mathrm{kV}$.

\section{Discussion}

During the desorption of noble gas implanted in silicon four activation energies have been observed $E_{\mathrm{A}}=1.25 \pm 0.05 \mathrm{eV}, E_{\mathrm{B}}=2.45 \pm 0.15 \mathrm{eV}, E_{\mathrm{C}}=$ $4.5 \pm 0.1 \mathrm{eV}$ and $E_{\mathrm{D}} \approx 10 \mathrm{eV}$. These values are almost the same for $\mathrm{Ne}, \mathrm{Ar}$ and $\mathrm{Kr}$ if the corresponding desorption mechanisms are present and are almost independent of the amount of noble gas collected. The influence of the noble gas arises in the distribution of the desorbed particle dose at the four mechanisms.

During the desorption of noble gas a linear decrease in the ellipsometric value $\delta \psi$ has been observed indicating a proportionality between damage recovery and noble gas desorption.

These observations support the conclusion that the diffusion mechanisms with respect to the activation energies have to be explained by the silicon properties and that the properties of the implanted noble gas are much less important. It is amazing that the values of the observed discrete activation energies form the first four terms of a geometrical series.

Desorption experiments of implanted $\mathrm{Si}(111)$ crystals with $\mathrm{He}, \mathrm{Ne}$ and $\mathrm{Kr}$ have also been performed by Davies and Carter [10], using a constant heating rate $\beta=30 \mathrm{~K} / \mathrm{s}$. The samples were bombarded at normal incidence at 400 and $1000 \mathrm{~V}$. Assuming the desorption mechanism can be described by a first-order desorption process with $p_{1}=10^{13} \mathrm{~s}^{-1}$, see eqs. (1) and (3), they obtained activation energies of $0.87(\mathrm{He}), 0.98(\mathrm{Ne})$ and $1.02 \mathrm{eV}(\mathrm{Kr})$ for the first desorption peak. Davies and Carter explain these values by an activation energy of $1.2-1.3 \mathrm{eV}$ for the migration of divacancies in silicon, as shown by EPR [14] and infrared absorption studies [15]. They assume, because the noble gas atoms do not participate in the covalent bonding, that a smaller activation energy is required if the divacancies will reorientate to the inert gas atom, causing motion of the atom, so they expect the activation energy to increase with the mass of the gas atom. Also they explain the broadening of the same desorption peaks at the high temperature side: "it is probable that some of the inherent gas atoms may be sufficiently remote from the surrounding divacancies to require normal divacancy motion for their release".

We only agree with the last quotation to explain the observed activation energy of peak $A$. If we adopt such an explanation then the observed $\nu_{1}=2 \times 10^{5} \mathrm{~s}^{-1}$ value for peak A can only be explained if the probability $\gamma$ to find a divacancy in the neighbouring sites, see eq. (22), will be $10^{-5}$. 
Furthermore, if we adopt the values for He of Davies and Carter, then at room temperature we can write, see eq. (1), for a first-order reaction: $\mathrm{d} N / \mathrm{d} t$ $=-N / \tau$ or $N=N_{0} \exp (-t / \tau)$ with $\tau=1 / \nu_{1} \exp (E / k T)$. With $E=0.87 \mathrm{eV}$, $\nu_{1}=10^{13} \mathrm{~s}^{-1}$ and $T=300 \mathrm{~K}$, the value of $\tau$ is $\sim 40 \mathrm{~s}$. So if one does not start the desorption experiment within $5 \tau \approx 200 \mathrm{~s}$ all gas will already have been released at room temperature. It becomes even worse if one considers the gas release during bombardment, because the collected ion dose will never exceed $\dot{N}_{\mathrm{b}} \tau \approx 10^{-4} \AA^{-2}$ if we use a value $\dot{N}_{\mathrm{b}}=2.5 \times 10^{10}$ ion $\mathrm{cm}^{-2} \mathrm{~s}^{-1}$, as typically used by Davies and Carter. This is in contradiction to their observation, that there is no significant reduction in the size of the spectrum if the implantation is performed at $80 \mathrm{~K}$ and the heating cycle starts immediately at $80 \mathrm{~K}$.

The largest desorption peak for all three noble gases ( $\mathrm{Ne}, \mathrm{Ar}, \mathrm{Kr}$ ) arises in the temperature range $850-900 \mathrm{~K}$, with activation energies $E_{\mathrm{B}}$ and $E_{\mathrm{C}}$. Our optical measurements, [1], have shown that in this temperature range the amorphous layer recrystallization is substantial. This suggests that during damage recovery the noble gas is "pushed" out of the sample, first with an activation energy $E_{\mathrm{B}}$ and afterwards with an activation energy $E_{\mathrm{C}}$. Because as soon as some damage recovery has been taken place the surroundings of the noble gas atoms change.

Hora [16] discussed the influence of mechanical stress on the activation energy, in a bombarded amorphous layer. For silicon a stress energy $E_{\mathrm{st}}$ of about 1-2 eV per atom can explain the increase of the diffusion coefficient $D=D_{0} \exp (-E / k T)$ in a crystal without stress to a value $D_{0} \exp [-(E-$ $\left.E_{\text {st }}\right) / k T$ ] in the crystal with stress. Also $E_{\text {st }}$ can be a function of coordinate or time. Furthermore if there is no stress between a crystalline silicon layer and a vacuum-deposited amorphous silicon layer the epitaxial regrowth can be described by an activation energy of $4.7 \mathrm{eV}$ [17], and corresponds to that for self-diffusion of silicon [18]. For amorphous silicon layers with stress, values of 2.35 up to $2.7 \mathrm{eV}$ are reported $[19,20]$.

The suggestion Hora made to explain the decrease of the activation energy of $E_{\mathrm{c}}$ to $E_{\mathrm{B}}$ by about $2 \mathrm{eV}$ being due to the influence of mechanical stress leads to some remarks. Firstly a compression of about $16 \%$ of amorphous silicon has to be assumed, independently of the amount of implanted noble gas. In an amorphous layer on top of a crystalline substrate with such a high compression an expansion must be expected, at least in a direction perpendicular to the sample. This has not been observed [1]. Secondly, because the stress will be reduced during damage recovery, it is not clear why investigators do not observe a continuous spectrum of activation energies between $E_{\mathrm{B}}$ and $E_{\mathrm{c}}$.

Finally peak D can be attributed to diffusion of noble gas in the "recrystallized" silicon, and occurs especially in the case of $\mathrm{Kr}$. Apparently an amount of noble gas remains which is not driven out at the most important damage recovery at $T \approx 880 \mathrm{~K}$. 


\section{Acknowledgements}

The RBS measurements where carried out in the "van de Graaff" laboratory in Utrecht. We would like to thank Dr. F.H.P.M. Habraken and Drs. J.B. Oude Elferink for their measurements with the RBS equipment.

\section{References}

[1] A.H.M. Holtslag, Noble Gas Ion Bombardment on Clean Silicon Surfaces, Studied Using Ellipsometry and Desorption. Thesis, Twente University of Technology (1986).

[2] P.A. Redhead, Vacuum 12 (1962) 203.

[3] G. Carter, Vacuum 12 (1962) 245.

[4] F.M. Lord and J.S. Kittelberger, Surface Sci. 43 (1974) 173.

[5] D.A. King, Surface Sci. 64 (1977) 43.

[6] E. Habenschaden and J. Kuppers, Surface Sci. 138 (1984) L147.

[7] P. Forzatti, M. Borghesi, I. Pasquon and E. Tronconi, Surface Sci. 137 (1984) 595.

[8] G. Busch and H. Schade, Lectures on Solid State Physics, Vol. 79 of International Series in Natural Philosophy (Pergamon Press, Oxford, 1976) p. 106.

[9] P.A. Redhead, J.P. Hobson and E.V. Kornelsen, The Physical Basis of Ultrahigh Vacuum, High Vacuum Series (Chapman \& Hall, London, 1968) table 2-27.

[10] A.L.M. Davies and G. Carter, Radiation Effects 10 (1971) 227.

[11] J. Yarwood, High Vacuum Technique, High Vacuum Series (Chapman \& Hall, London, 1967) ch. 1.

[12] M. Wutz, H. Adam and W. Walcher, Theorie and Praxis der Vakuumtechnik (Vieweg, Braunschweig, 1981).

[13] F. Schulz and K. Wittmaack, Radiation Effects 29 (1975) 31.

[14] G.D. Watkins and J.W. Corbett, Phys. Rev. A138 (1965) 543.

[15] L.J. Cheng, J.C. Corelli, J.W. Corbett and G.D. Watkins, Phys. Rev. 152 (1966) 761.

[16] H. Hora, Appl. Phys. A32 (1983) 217.

[17] B.Y. Tsaur and L.S. Hung, Appl. Phys. Letters 37 (1980) 648.

[18] R.N. Ghoshtagore, Phys. Rev. Letters 16 (1966) 890.

[19] A. Lietoila, R.B. Gold and J.F. Gibbons, Appl. Phys. Letters 39 (1981) 810.

[20] H. Nishi, T. Sakurai and T. Furuya, J. Electrochem. Soc. 125 (1978) 461.

[21] J.A. Venables, G.D.T. Spiller and M. Hanbucken, Rept. Progr. Phys. 47 (1984) 399. 